\title{
Allergic fungal rhinosinusitis infiltrating anterior skull base and clivus
}

\author{
Giuseppe Meccariello ${ }^{a}$, Alberto Deganello ${ }^{a}$, Giuditta Mannelli ${ }^{a}$, Giacomo Bianco ${ }^{\mathrm{a}}$, Franco Ammannati ${ }^{\mathrm{b}}$, \\ Christos Georgalas ${ }^{c}$, Oreste Gallo ${ }^{\mathrm{a}, *}$
}

${ }^{\mathrm{a}}$ Academic Clinic of Otolaryngology-Head and Neck Surgery, University of Florence, Italy
${ }^{\mathrm{b}}$ Neurosurgery Unit, Azienda Ospedialiero-Universitaria Careggi, Florence, Italy

${ }^{\mathrm{c}}$ Endoscopic Skull Base, Amsterdam Medical Center, Amsterdam, The Netherlands

\section{A R T I C L E I N F O}

\section{Article history:}

Received 3 February 2012

Accepted 29 June 2012

Available online $\mathrm{xxx}$

\section{Keywords:}

AFRS

Bone erosion

Skull base

Clivus

Eosinophil

Rhinosinusitis

\begin{abstract}
A B S T R A C T
Bone erosion and skull base invasion are often suggestive of a malignant mass in paranasal and nasal cavities. Nevertheless, forms of chronic rhinosinusitis, such as allergic fungal rhinosinusitis (AFRS), could mimic malignant features. Here, we report AFRS patient with orbital, anterior cranial fossa, Turkish saddle and clivus erosion. A 48-year-old Caucasian female with history of drug-resistant headache, nasal obstruction and anosmia was referred to our institution. Imaging showed hyperdense featureless tissue with signs of medial orbital wall, cribiform lamina and clivus erosions and encasement of right internal carotid artery. Massive amounts of thick and grayish mucoid material were evacuated during surgery. In case of bony erosion, malignancy should always be excluded. Often the correct diagnosis will be obtained only by operative specimens. AFRS could usually be managed endoscopically. Appropriate medical management of the AFRS should be administered in order to prevent relapses.
\end{abstract}

(c) 2012 Elsevier Ireland Ltd. All rights reserved.

\section{Introduction}

A bone-eroding mass in the nasal cavity and/or paranasal sinuses should be always investigated in order to exclude a malignancy. It is important to note however that a chronic, nontissue invasive, inflammatory process of the nose and paranasal sinuses, the so called allergic fungal rhinosinusitis (AFRS), could mimic neoplastic features. It represents an immune hypersensitivity to extramucosal fungal antigens, which leads to the production and accumulation of allergic mucin. The presentation of AFRS is usually subtle, consisting of gradually increasing nasal obstruction, nasal crusting, viscous rhinorrea, and hyposmia. Unlike bacterial rhinosinusitis, facial or dental pain and headaches are less common [1,2]. Conversely, multiple nasal polyps are virtually identified in all AFRS patients, with unilateral distribution more common than bilateral [2-4]. The classic and still widely accepted diagnostic criteria for AFRS were described by Bent and Kuhn [3], who proposed the following: type 1 hypersensitivity; nasal polyposis; characteristic computed tomography (CT) scan findings; eosinophilic mucus without fungal invasion into sinus tissue; and a positive fungal stain of evacuated sinus contents. The

\footnotetext{
* Corresponding author at: Academic Clinic of Otolaryngology-Head and Neck Surgery, University of Florence, via Largo Brambilla, 3-50134 Firenze, Italy. Tel.: +39 0557947988; fax: +39 0557947939 .

E-mail address: o.gallo@med.unifi.it (O. Gallo).
}

disease's course is typically indolent and non-aggressive; however, as allergic mucin accumulates within the paranasal sinuses, it may form a mucocele and mimic an invasive process. In $6-56 \%$ of patients with AFRS, the expanding mass leads to bony erosion and involvement of adjacent structures [5]. However, investigators soon noted that in some cases, the allergic mucin evacuated from the sinuses did not have identifiable fungal elements; these patients were labeled as having an "AFRS-like syndrome" [6]. Additionally, Ferguson [4] proposed the term "eosinophilic mucin rhinosinusitis" (EMRS) to describe cases in which fungus was not identified histologically. Moreover, she demonstrated that although AFRS and EMRS had identical physical findings, there were clinical differences such as acetylsalicylic acid (ASA) sensitivity was more common in EMRS cases as well as the sinus disease was exclusively bilateral and asthma was present in nearly all EMRS patients.

The primary management for both clinical entities was represented by surgery. The goals of the surgery are:

1. to clear out the edematous mucosa,

2. to create patent sinus ostia large enough to facilitate adequate drainage and ventilation,

3. to marsupialize involved sinuses.

Although effective at removing large portions of the disease, surgery is insufficient in eradicating the relentless inflammation of AFRS. In the absence of medical adjuvant therapy, recurrence rates

0385-8146/\$ - see front matter (c) 2012 Elsevier Ireland Ltd. All rights reserved. http://dx.doi.org/10.1016/j.anl.2012.06.005 
of up to $100 \%$ have been reported [2,7]. Here, we report a case of an AFRS with anterior skull base and clivus involvement treated by functional endoscopic sinus surgery (FESS).

\section{Case report}

A 48-year-old Caucasian female referred to our institution with a month history of drug-resistant headache, nasal obstruction and anosmia. Her past medical history was significant for ASA sensitivity and asthma. Sinonasal endoscopy revealed many large polyps, occluding the nasal cavities, no muco-purulent discharge nor post nasal dripping as well as no neck masses nor pathologic lymph nodes were found. The patient underwent a CT scan that showed hyperdense formless tissue involving all paranasal sinuses with signs of medial orbital wall erosion, reduction of ethmoidal cells thickness, massive encasement of right internal carotid artery in sphenoid sinus, erosion of the cribiform lamina and clivus (Fig. 1). A contrast-enhanced magnetic resonance imaging (MRI) was performed in order to clarify the extension of the mass and to visualize any hypervascularization. This heterogeneous mass did not appear to invade the dura, the pituitary gland, the carotid and basilar arteries; furthermore, a peripheral contrast-enhancement was noted (Fig. 2). The patient underwent an endoscopic drainage, with the neurosurgical team on standby in case an open neurosurgical approach was required. As first step biopsies of nasal polyps were obtained and intraoperatively examined. Inflammatory tissue was shown in all specimens, therefore we performed wide openings of all paranasal sinuses removing a dense and grayish mucin that revealed an intact paranasal mucosa. The intraoperative examination of the mucin demonstrated few eosinophilic cells without any fungal hyphae. This tenaceous material fulfilled each paranasal sinuses involving the medial orbital wall at both sides; after its complete removal in sphenoid sinus, a right internal carotid and optic nerve dehiscences were found. Further, frontal disease was cleared. The operation was free of complications and open approaches were not required. The subsequent pathology report (Fig. 3) indicated that no fungal elements were visualized within the tissue; the mucin was described as an amorphous mucoid material with an extensive collection of eosinophils and neutrophils, Charcot-Leyden crystals were not identified and fungal staining and cultures were negative. Serum levels of immunoglobulins showed low IgE $(238 \mathrm{mg} / \mathrm{dL})$ and a IgG deficiency $(523 \mathrm{mg} / \mathrm{dL})$ especially for $\operatorname{IgG} 1(317 \mathrm{mg} / \mathrm{dL})$. The patient was discharged with topic corticosteroid therapy and saline irrigations. At the 3-month follow-up, the patient had healed completely, the paranasal sinus ostia were well opened with excellent functional results and no mucin was present. All of her rhinosinusitis-symptoms had resolved.

\section{Discussion}

In AFRS-suffering patients, multiple nasal polyps are identified with unilateral distribution more common than bilateral [2-4]. Further, in $6-56 \%$ of AFRS patients, the expanding mass leads to bony erosion and involvement of adjacent structures [5]. Although the erosion may be extensive, it is important to note that the disease process itself does not invade tissues. Some postulate that the expanding mucin exerts pressure on neighboring mucosal blood vessels, thus compromising blood supply to the underlying bone. Ischemia ensues, weakening bony structures, which become increasingly susceptible to mechanical stress and necrosis [8]. The location of the disease, as well as the path of least resistance, will determine where expansion occurs. Owing to its innate weakness, the lamina papyracea is the most common location for bony destruction and the orbit is the most common location for extrasinus disease spread. When allergic mucin extends into the orbit, the patient often presents with ocular signs ranging from proptosis to visual field loss [5]. In advanced presentations, lesions may erode the skull base, leading to intracranial presence of mucin.

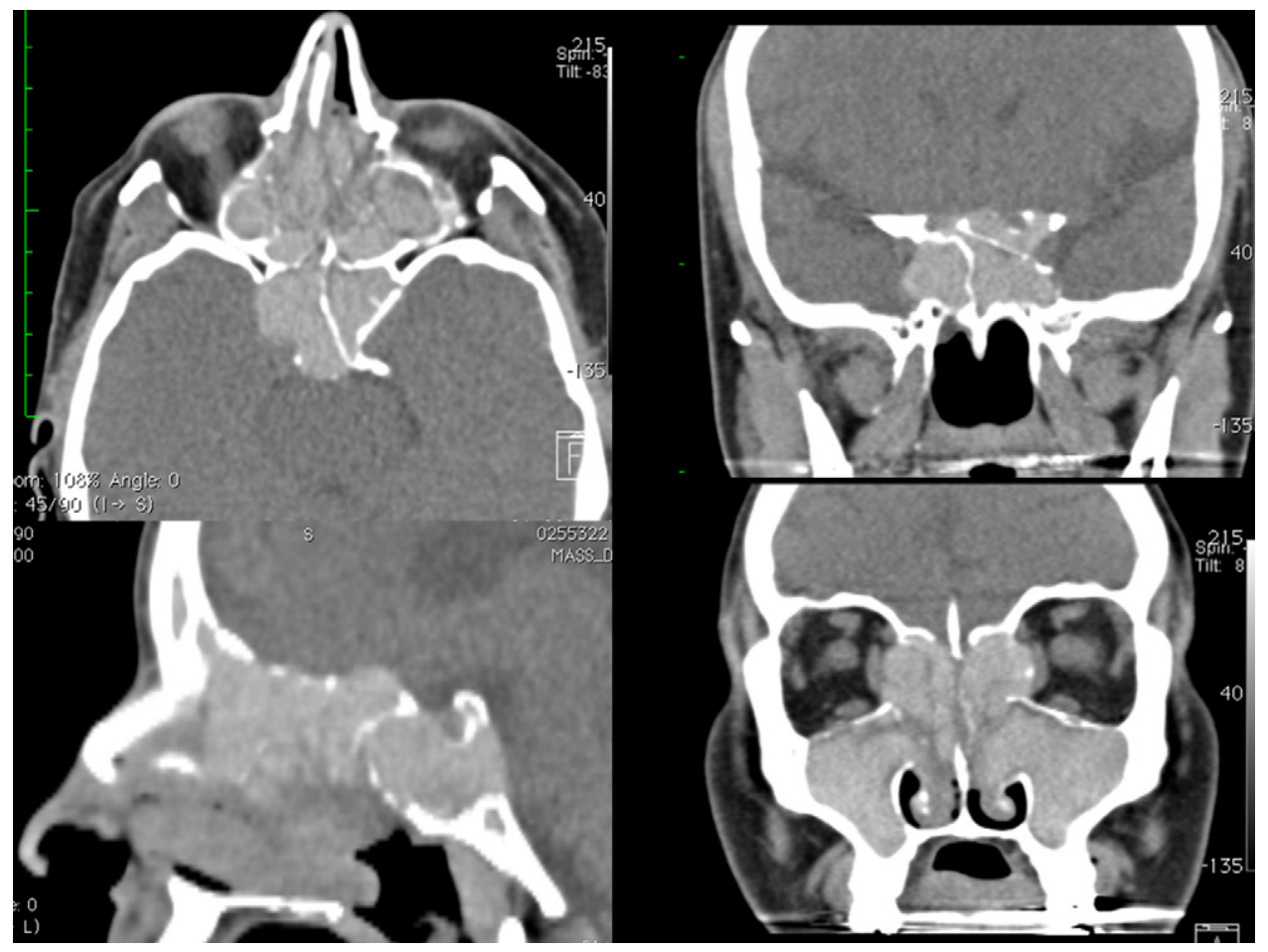

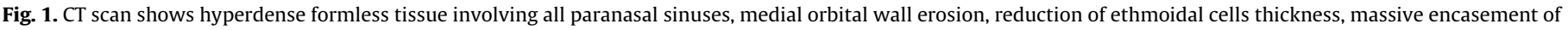
right internal carotid artery in sphenoid sinus, erosion of the cribiform lamina and clivus.

Please cite this article in press as: Meccariello G, et al. Allergic fungal rhinosinusitis infiltrating anterior skull base and clivus. Auris Nasus Larynx (2012), http://dx.doi.org/10.1016/j.anl.2012.06.005 


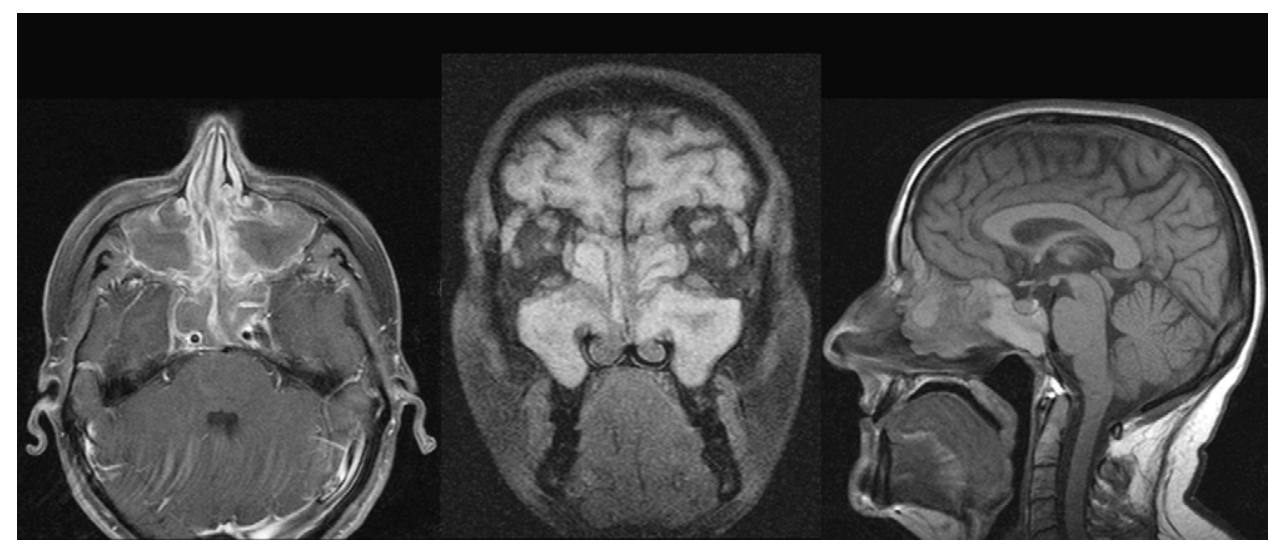

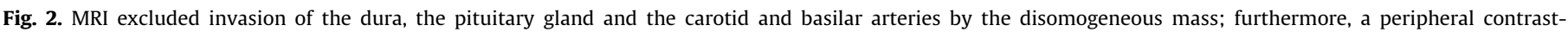
enhancement was highlighted.

Intracranial extension may become complicated with headaches, frontal lobe compression, facial deformity, or even an intracranial abscess [8]. Initial AFRS management planning includes radiographic imaging. Noncontrast paranasal sinus CT is the preferred modality, which demonstrates irregular, hyperdense masses involving multiple sinuses. The most commonly involved are the ethmoid sinus, followed by the maxillary, frontal, and sphenoid sinuses. The opacified sinuses often exhibit small areas of heterogeneous hyperattenuation, which appear as dots or streaks in soft tissue windows. These areas of hyperattenuation are believed to represent accumulated calcium and heavy metal salts within the allergic mucin. It is possible that fungal elements act as a nidus for precipitation of the salts. When CT fails to confirm a clinical picture of AFRS, MRI may be a useful alternative. MRI reveals masses with decreased or void intensities on T1- and T2weighted images, respectively, with sinus mucosa appearing enhanced [8]. Although CT and MRI findings are characteristic of dense extramucosal mucin, they are not specific to AFRS and can provide only a presumptive diagnosis. For a definitive diagnosis of AFRS, samples of mucin and mucosa must be obtained. It is essential that specimens be acquired surgically and not with an "office swab," which will grow floral fungi from virtually any nose [9]. Histopathologic preparations show mucus peppered with Charcot-Leyden crystals and eosinophils with variable numbers of fungal elements, which are best visualized using fungal stains. Cultures of the mucin mostly grow dermatiaceous fungi such as Alternaria, Bipolaris, and Curvularia species [3,6,9]. Under the microscope, sinus mucosal sections demonstrate no evidence of necrosis, granulomatosis, or fungal invasion $[2,9]$. However, lacks evidence of a fungal presence has been reported [4,6]. Ferguson conducted a retrospective and prospective review of 69 such cases and coined the idea of EMRS [4]. She found that although AFRS and EMRS had identical physical findings, there were statistically significant clinical differences in both groups. The mean age of AFRS patients was younger (30.7 years) than that of EMRS patients (48.0 years). Asthma was present in nearly all EMRS patients (93\%) and about half of AFRS patients (41\%). ASA sensitivity was much more common in EMRS cases (54\%) than AFRS cases (14\%). Sinus disease was unilateral in 55\% of AFRS cases but exclusively bilateral in EMRS. Total IgE levels were consistently higher for AFRS patients, and nasal polyps were present in nearly all AFRS and EMRS patients. These differences suggest that AFRS is likely driven
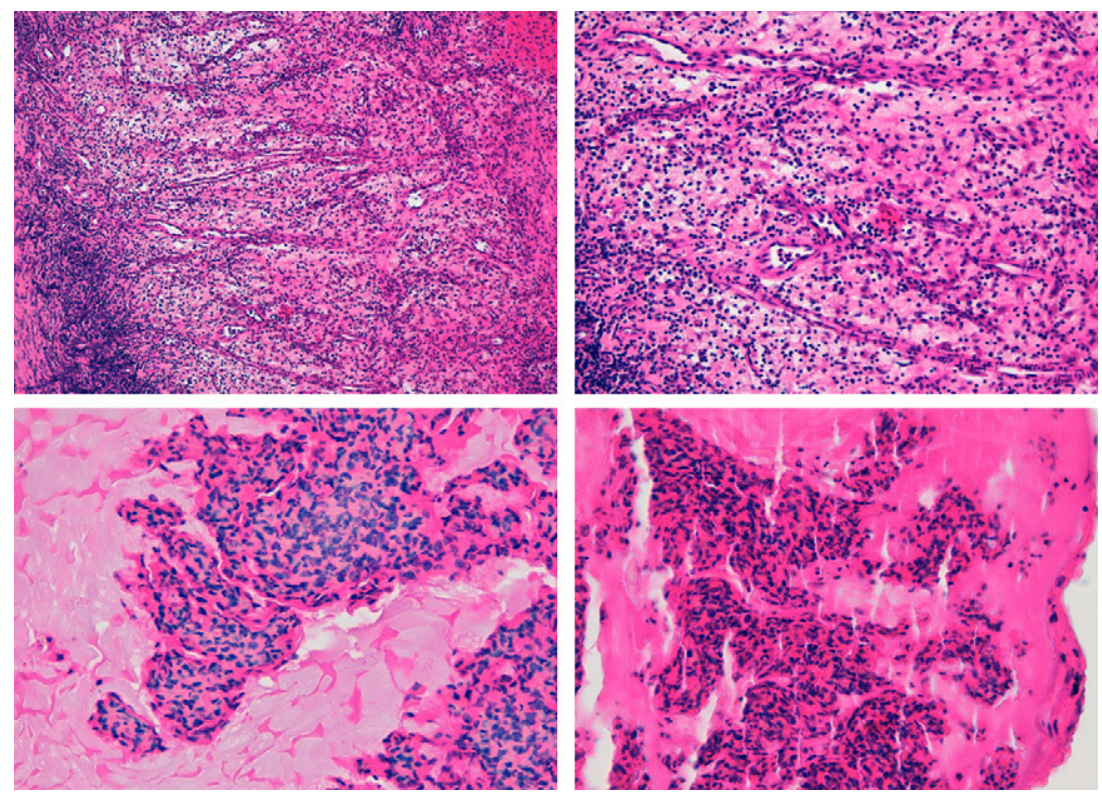

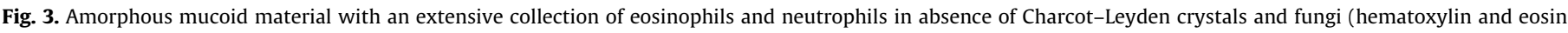
staining; $2.5 \times$ and $5 \times$ ).

Please cite this article in press as: Meccariello G, et al. Allergic fungal rhinosinusitis infiltrating anterior skull base and clivus. Auris Nasus Larynx (2012), http://dx.doi.org/10.1016/j.anl.2012.06.005 
by a localized IgE-mediated reaction to fungal antigens, whereas EMRS occurs because of systemic immunologic deregulation. The idea that EMRS is a systemic entity could explain the high association with lower airway and bilateral paranasal sinus pathology. Nevertheless we feel that differences between AFRS and EMRS are too few to justify the codification of a new clinical entity. Our patient presented some clinical features that satisfied Ferguson's criteria for EMRS [4]: the patient suffered asthma with ASA intolerance, was older than 40 years of age and sierologic analysis demonstrated low levels of IgE with low levels of IgG subclasses, showed small areas of heterogeneous hyperattenuation at MRI (Fig. 2). Nevertheless only the term AFRS has been used in recent review of chronic sinusitis, and furthermore the presence of extra sinus disease extension shown by our patient is not listed within Ferguson's requirements for EMRS [10-13]. Despite the lack of randomized controlled trials, it is accepted that the treatment of AFRS and must include surgical decompression of the sinuses, nasal saline irrigation, and corticosteroid therapy, either systemic or local. The best clinical outcomes might be achieved in this way. In our case surgical treatment with functional endoscopic surgery followed by saline irrigations and topic administration of corticosteroids proved to be adequate.

Although effective in removing large portions of the disease, surgery is insufficient in eradicating the relentless inflammation of AFRS. In the absence of medical adjuvant therapy, recurrence rates of up to $100 \%$ have been reported [2,7]. Unfortunately, randomized controlled trials have yet to determine optimal doses, duration, and timing of corticosteroid therapy and whether either oral or inhalant corticosteroids have different outcomes. In addition to inflammation, atopy and antigen exposure have been targets of adjuvant therapy. To decrease antigen exposure, two options are available: irrigation and topical antifungals. Nasal saline irrigation is very safe and known to be effective in promoting nasal ciliary clearance reducing intranasal inflammatory cytokines; thus, it is recommended as part of AFRS treatment regimen. Since Bent and Kuhn [14] demonstrated that fungi, found in AFRS, can be susceptible to antifungal agents in vitro, antifungal systemic medications have also been administered in AFRS patients; however, their utility remains rather controversial in extramucosal fungal diseases since convincing supportive data are still missing [2,15].

Finally, despite AFRS could mimic a malignant tumor of paranasal sinuses with extensive bony erosion encasing vital structures, the endoscopic approach allows to achieve reasonable functional results removing all pathologic material and obtaining full aerated sinuses. It is crucial to exclude any malignant or invasive fungal disease, therefore mucosal samples should be intraoperatively analyzed. Although in the majority of cases the solely endoscopic approach is adequate, a neurosurgical team should be alerted since an intraoperative diagnosis of malignancy or invasive fungal disease could require a switch to a combined endoscopic/open surgical approach in order to achieve clear resection margins and a suitable reconstruction.

\section{Conclusion}

A bone erosing nasal/paranasal sinus mass should always investigated to exclude a malignancy, although a chronic inflammatory process could be assumed. However, the definitive diagnosis of AFRS rests in intraoperative analysis, it is important to recognize the entity in a clinical setting. Patients presenting with rhinosinusitis-like symptoms who demonstrate patches of hyperattenuation in a background of mucoceles on CT images should be prime suspects for AFRS. Once a suspicious of chronic inflammatory process strongly presumed, the endoscopic surgical treatment to decompress nasal and paranasal cavities should be performed. In rare cases, an intracranial neurosurgical team approach could be necessary to extirpate mucin and to reconstruct bone defects. Although surgery is very effective, without adjuvant treatment, recurrence is almost guaranteed. Both oral and inhalant corticosteroids have a pivotal role whilst antifungal drugs seems to have conflicting results. Therefore further investigation with randomized control trials will be necessary.

\section{Conflict of interest}

All authors disclose any financial and personal relationships with other people or organizations that could influence the own work.

\section{References}

[1] Manning SC, Holman M. Further evidence for allergic pathophysiology in allergic fungal sinusitis. Laryngoscope 1998;108:1485-96.

[2] Marple BF. Allergic fungal sinusitis: current theories and management strategies. Laryngoscope 2001;111:1006-19.

[3] Bent JP, Kuhn FA. Diagnosis of allergic fungal sinusitis. Otolaryngol Head Neck Surg 1994;111:580-8.

[4] Ferguson BJ. Eosinophilic mucin rhinosinusitis: a distinct clinicopathological entity. Laryngoscope 2000;110:799-813.

[5] Ghegan MD, Lee FS, Schlosser RJ. Incidence of skull base and orbital erosion in allergic fungal rhinosinusitis (AFRS) and non-AFRS. Otolaryngol Head Neck Surg 2006;134:592-5

[6] Cody II DT, Neel III HB, Ferreiro JA, Roberts GD. Allergic fungal sinusitis: the Mayo Clinic experience. Laryngoscope 1994;104:1074-9.

[7] Saravanan K, Panda NK, Chakrabarti A, Das A, Bapuraj RJ. Allergic fungal rhinosinusitis: an attempt to resolve the diagnostic dilemma. Arch Otolaryngol Head Neck Surg 2006;142:173-8.

[8] Nussenbaum B, Marple BF, Schwade ND. Characteristics of bony erosion in allergic fungal rhinosinusitis. Otolaryngol Head Neck Surg 2001;124:150-4.

[9] Taylor MJ, Ponikau JU, Sherris DA, Kern EB, Gaffey TA, Kephart G, et al. Detection of fungal organisms in eosinophilic mucin using a fluorescein-labeled chitinspecific binding protein. Otolaryngol Head Neck Surg 2002; 127:377-83.

[10] Hutcheson PS, Schubert MS, Slavin RG. Distinctions between allergic fungal rhinosinusitis and chronic rhinosinusitis. Am J Rhinol Allergy 2010;24:405-8.

[11] Hsu J, Peters AT. Pathophysiology of chronic rhinosinusitis with nasal polyp. Am J Rhinol Allergy 2011;25:285-90.

[12] Hamilos DL. Chronic rhinosinusitis: epidemiology and medical management. J Allergy Clin Immunol 2011:128:693-707.

[13] Ryan MW. Allergic fungal rhinosinusitis. Otolaryngol Clin North Am 2011; 44:697-710.

[14] Bent III JP, Kuhn FA. Antifungal activity against allergic fungal sinusitis organisms. Laryngoscope 1996;106:1331-4.

[15] Ponikau JU, Sherris DA, Weaver A, Kita H. Treatment of chronic rhinosinusitis with intranasal amphotericin B: a randomized, placebo-controlled, doubleblind pilot trial. J Allergy Clin Immunol 2005;115:125-31. 